\title{
Experience from a Medical Students Leadership Workshop
}

\author{
Muhamad Saiful Bahri Yusoff, Ahmad Fuad Abdul Rahim \& Mohd Najib Mat Pa
}

Medical Education Department, School of Medical Sciences, Universiti Sains Malaysia.

\section{Context \\ Positive personal qualities, soft skills and professional development were realized as important elements in our school's curriculum since its inception 30 years ago. Relevant inputs were imparted to students at various places in the time table. In 2007, the Student Soft Skills Development Programme was formed to facilitate and coordinate training programmes in such areas in the undergraduate. We describe in this article a leadership development programme known as the 'Medical Students Leadership Workshop' in our medical school.}

\section{Reason for the idea}

The importance of promoting and nurturing positive personal qualities and professional development among future doctors are becoming more apparent within healthcare. Many evidences showed that it has effects on the quality of care provided. This workshop was developed to enhance students' self-awareness of their leadership potential and to assist them in developing self-improvement strategies to develop their leadership skills. It offers a unique opportunity to develop students' skills in such areas.

\section{Methods}

The workshop was run over one half-day. It was limited to 20 participants per workshop. The same workshop will be conducted for several times to cater for different groups of medical students.

The workshop was run by academic staff from the Medical Education Department. The objectives of this workshop were to enable students to indentify and explore their leadership styles using the CREDO Personality Profile (CPP), to understand the importance of leadership styles and to apply their personal leadership styles for becoming better medical students as well as future doctors. Participants were given some input regarding the importance of leadership styles related to medical study as well as in the medical career. Discussion sessions were held to explore each leadership style further. After knowing and understanding their personal leadership styles, students should be able to recognize their own strengths and weaknesses and develop their own selfimprovement strategy to strengthen their leadership skills development in the future.

\section{Evaluation}

A total of 20 medical students participated in the workshop. At the end of the workshop, evaluation questionnaires were distributed to participants. Apart from basic biographic data, the questionnaire solicited participants' ratings regarding, the usefulness of input given, facilitators and the discussion sessions. Participants were also asked to rate the success of the workshop in achieving the objectives and its overall usefulness. In an open-ended section participants were asked to describe the most important thing they learnt from the workshop as well as the most important thing that can be improved. Data analysis was done using SPSS version 12. The evaluation showed that the participants rated the overall workshop as highly useful (87.1\%) and as having achieved the objectives $(85 \%)$. All sessions were rated as highly useful. Among the comments written in the open-ended section regarding the most important thing participants learnt from the workshop, $55.6 \%$ wrote comments related to improved self-awareness. The importance of leadership styles was commented by $22.2 \%$ of them. Another $22.2 \%$ appreciated the usefulness of increased knowledge such as leadership qualities and styles.

Corresponding Author: Dr Muhamad Saiful Bahri bin Yusoff, Lecturer, Department of Medical Education, School of Medical Sciences, Universiti Sains Malaysia, 16150 Kubang Kerian, Kelantan, Malaysia.

Email:msaiful@kb.usm.my 